\title{
Short-Chain Fatty Acids Manifest Stimulative and Protective Effects on Intestinal Barrier Function Through the Inhibition of NLRP3 Inflammasome and Autophagy
}

\author{
Yanhai Feng $^{\mathrm{a}} \quad$ Yu Wang ${ }^{\mathrm{b}} \quad$ Pei Wang ${ }^{\mathrm{a}} \quad$ Yalan Huang ${ }^{\mathrm{c}} \quad$ Fengjun Wang $^{\mathrm{a}}$ \\ aState Key Laboratory of Trauma, Burns, and Combined Injury, Institute of Burn Research, Southwest \\ Hospital, Third Military Medical University (Army Medical University), Chongqing, ${ }^{b}$ Department of \\ Gastroenterology, Southwest Hospital, Third Military Medical University (Army Medical University), \\ Chongqing, China 'Department of Military Nursing, School of Nursing, Third Military Medical University \\ (Army Medical University), Chongqing, China
}

\section{Key Words}

Short-chain fatty acids - Intestinal barrier - Tight junction - Lipopolysaccharide NLRP3 inflammasome $\cdot$ Autophagy

\begin{abstract}
Background/Aims: Short-chain fatty acids (SCFAs) are the major energy resources of intestinal epithelial cells. It has been reported that SCFAs can repair the dysfunction of intestinal barrier, however, the underlying mechanisms are still not fully understood. Here, we investigated the stimulative and protective effects of SCFAs on intestinal barrier function and the possible mechanisms. Methods: To investigate the effects of SCFAs on intestinal barrier function, the Caco-2 monolayers were exposed to acetate, propionate, butyrate respectively or simultaneously without or with lipopolysaccharide (LPS). Next, Caco-2 cells were treated with trichostatin A and etomoxir to identify whether SCFAs act as HDAC inhibitors or energy substances. To activate NLRP3 inflammasome and autophagy, Caco-2 cells were treated with LPS+ATP and rapamycin respectively without or with SCFAs. The transepithelial electrical resistance (TER) and paracellular permeability were respectively detected with a MillicellERS voltohmmeter and fluorescein isothiocyanate-labeled dextran. Immunoblotting and immunofluorescence were applied to analyze the expression and distribution of tight junction proteins, and the activation of NLRP3 inflammasome and autophagy. Results: Acetate (0.5mM), propionate $(0.01 \mathrm{mM})$ and butyrate $(0.01 \mathrm{mM})$ alone or in combination significantly increased TER, and stimulated the formation of tight junction. SCFAs also dramatically attenuated the LPS-induced TER reduction and paracellular permeability increase, accompanying significantly

\footnotetext{
Fengjun Wang State Key Laboratory of Trauma, Burns and Combined Injury, Institute of Burn Research, Southwest Hospital Third Military Medical University, 30 Gaotanyan Street, Chongqing, 400038 (China) Tel. +86-23-68754176; Fax 86-23-65460398; E-Mail wangfj@tmmu.edu.cn
} 
alleviated morphological disruption of ZO-1 and occludin. Meanwhile, the activation of NLRP3 inflammasome and autophagy induced by LPS were significantly inhibited by SCFAs. Trichostatin A imitated the inhibiting action of SCFAs on NLRP3 inflammasome, whereas etomoxir blocked the action of SCFAs on protecting intestinal barrier and inhibiting autophagy. In addition, the activation of autophagy and NLRP3 inflammasome by rapamycin and LPS+ATP resulted in TER reduction, paracellular permeability increase and morphological disruption of both ZO-1 and occludin, which was alleviated by SCFAs. Conclusion: It is suggested that SCFAs stimulate the formation of intestinal barrier, and protect the intestinal barrier from the disruption of LPS through inhibiting NLRP3 inflammasome and autophagy. In addition, SCFAs act as energy substances to protect intestinal barrier and inhibit autophagy, but act as HDAC inhibitors to suppress NLRP3 inflammasome. Furthermore, the mutual promoting action between NLRP3 inflammasome and autophagy would destroy intestinal barrier function, which could be alleviated by SCFAs.

(C) 2018 The Author(s)

Published by S. Karger AG, Basel

\section{Introduction}

A primary function of intestinal epithelia is to form a biological barrier and maintain homeostasis. Tight junction and its associated proteins, including zonula occludens (ZO), occludin and claudins, are vital to intestinal epithelial paracellular barrier, which prevent luminal antigens or bacteria from entering into mucosa and contacting with the immune system [1-3]. However, it has been well documented that the disruption of intestinal epithelial barrier is compromised in a variety of intestinal diseases, including inflammatory bowel disease and Crohn's disease $[4,5]$. Thus, to protect intestinal barrier from pathological disruption is of great significance.

Short-chain fatty acids (SCFAs), the byproducts of dietary fiber in intestine during fermentation, are the most important energy substances of the epithelial lining of the colon [6]. Besides, abundant experiments have also indicated that butyrate, an important member of SCFAs, can protect intestinal barrier function by up-regulating tight junction protein claudin-1 or facilitating tight junction assembly [7, 8]. Furthermore, SCFAs are also well known as histone deacetylase (HDAC) inhibitors, and HDAC inhibitors such as trichostatin A can suppress inflammatory response $[9,10]$. Therefore, to further confirm the protective functions of SCFAs on intestinal barrier and its possible mechanisms is necessary.

NLRP3, the best characterized member of the NLRP sub-family, has been shown to trigger IL-1 $\beta$ and IL-18 processing and release in response to a variety of pathogens and endogenous dangerous signals including adenosine triphospate (ATP) and silica [11]. Activation of NLRP3 leads to recruitment of the adaptor protein ASC and of pro-caspase-1 to form a multiprotein complex termed the inflammasome. Then, inflammasome activation stimulates the caspase-1-dependent processing of inflammatory molecules pro-IL-1 $\beta$ and pro-IL-18, resulting in the secretion of the mature form of these two cytokines [12]. Meantime, it has been well illustrated that ROS production is closely associated with the activation of NLRP3 inflammasome [13]. Furthermore, it has been reported that NLRP3 inflammasome plays an important role in maintaining intestinal homeostasis $[11,14]$. However, the precise relationship between NLRP3 inflammasome and intestinal barrier function requires to be clarified.

Autophagy, an intracellular degradation pathway, refers to the engulfment and processing of cellular proteins, including damaged organelles and long-lived and misfolded proteins. As an essential cell survival mechanism, autophagy plays an important role in diverse processes such as metabolic stress and inflammatory diseases [15]. It has been documented that energy metabolism and ROS production are closely associated with autophagy activation $[16,17]$. Autophagy has also been shown to be active in the normal colonic intestinal mucosa, playing a role in intestinal cell survival during physiological stress [18]. Although autophagy can enhance intestinal barrier function [19], the exact relationship between autophagy and intestinal barrier function is still not fully determined. 


\section{Cellular Physiology Cell Physiol Biochem 2018;49:190-205 \begin{tabular}{ll|l|l} 
DOI: 10.1159/000492853 & $\begin{array}{l}\text { O } 2018 \text { The Author(s). Published by S. Karger AG, Basel } \\
\text { www.karger.com/cpb }\end{array}$ \\
and Biochemistry Published online: 22 August 2018
\end{tabular} \\ Feng et al.: Short-Chain Fatty Acids Protect Intestinal Barrier}

The aim of this study was to examine the role of SCFAs on regulation of intestinal barrier function, employing a commonly used in vitro intestinal epithelial model consisting of filter-grown Caco-2 monolayers. Our data indicate that SCFAs stimulate the formation of intestinal barrier function, and protect intestinal barrier from the disruption induced by LPS through inhibiting the activation of NLRP3 inflammasome and autophagy. Meanwhile, SCFAs act as energy substances to protect intestinal barrier and inhibit autophagy, but act as HDAC inhibitors to suppress NLRP3 inflammasome. Furthermore, NLRP3 inflammasome and autophagy could destroy intestinal barrier function respectively, and each of them plays interactive role with the other.

\section{Materials and Methods}

\section{Cell culture and monolayer preparation}

Caco-2 cells were purchased from American Type Culture Collection (Manassas, VA) and grown in DMEM supplemented with $4.0 \mathrm{mmol} / \mathrm{L}$ L-glutamine, $1 \%$ non-essential amino acids, $100 \mathrm{U} / \mathrm{ml}$ penicillin, 100 $\mathrm{U} / \mathrm{ml}$ streptomycin and $15 \%$ fetal bovine serum. The cells were subcultured by partial digestion with $0.25 \%$ trypsin and $0.53 \mathrm{mmol} / \mathrm{L}$ EDTA in Hank's balanced saline solution (HBSS) without $\mathrm{Ca}^{2+}$ and $\mathrm{Mg}^{2+}$. Caco-2 cells were seeded at $0.5 \times 10^{5} / \mathrm{cm}^{2}$ and grown as monolayers on collagen-coated permeable polycarbonate membrane Transwell supports with $0.4 \mu \mathrm{m}$ pores (Corning, Corning, NY), and used for experiments after confluence. Transwell supports with $0.33-\mathrm{cm}^{2}$ surface areas were used for electrophysiological studies.

\section{Treatments of Caco-2 monolayer}

In the experiments, acetate (Sigma, MO), propionate (Sigma), butyrate (Sigma), lipopolysaccharide (Sigma), rapamycin (Millipore, MA), adenosine 5-Triphosphate (Sigma), N-Acetyl-L-cysteine (Sigma), trichostatin A (Sigma) and etomoxir (Sigma) were added to the basal and apical chamber of Transwell supports. Caco-2 monolayers in control group were only incubated with DMEM withoutadditional treatment. In the experiment about the effect of SCFAs on intestinal barrier formation, the monolayers were cultivated for 24 hours before SCFAs were added. Then, transepithelial electrical resistance (TER) was measured for four consecutive days. For other experiments, the monolayers were cultivated for 10 days before diverse agents were added, and TER was detected for 24 consecutive hours.

\section{Measurement of TER}

TER values of all monolayers were measured with a Millicell-ERS voltohmmeter (Millipore, Bedford, MA) as described previously [20]. In order to facilitate comparisons between conditions, TER was normalized to initial value, and expressed as percentage of initials.

\section{Paracellular permeability assay}

The apical-to-basal paracellular flux of $4 \mathrm{kDa}$ fluorescein isothiocyanate-labeled dextran (FITCdextran, Sigma) across Caco-2 monolayers was assayed as described previously [20]. Briefly, at the end of

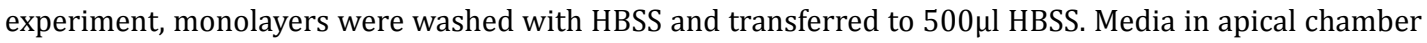
were gently aspirated and replaced with $100 \mu \mathrm{l}$ of $1 \mathrm{mg} / \mathrm{ml} \mathrm{FITC-dextran} \mathrm{dissolved} \mathrm{in} \mathrm{HBSS.} \mathrm{Then,} \mathrm{monolayers}$ were incubated at $37^{\circ} \mathrm{C}$ for 2 hours. The samples coming from basal chamber were used for fluorescence determination using a fluorescent microplate reader (Varioskan Flash, Thermo Electron Corporation, Vantaa, Finland) with an excitation wavelength of $480 \mathrm{~nm}$ and an emission wavelength of $520 \mathrm{~nm}$. FITCdextran flux was normalized to control.

\section{Immunoblotting analysis}

Caco-2 cells grown on $5.0-\mathrm{cm}^{2}$ Transwell supports were washed with ice-cold PBS, and lysed with Laemmli sample buffer $(50 \mathrm{mmol} / \mathrm{L}$ Tris- $\mathrm{HCl}, 2 \%$ SDS, $0.1 \%$ bromophenol blue, $5 \% \beta$-mercaptoethanol, $10 \%$ glycerine, $\mathrm{pH}$ 6.80), followed by a brief sonication with a sonicator (Tomy Seiko, Tokyo, Japan). Cell lysates were centrifuged at $10000 \mathrm{~g}$ for $10 \mathrm{~min}$ at $4{ }^{\circ} \mathrm{C}$ and heated at $100^{\circ} \mathrm{C}$ for $5 \mathrm{~min}$. Proteins were separated on $6 \%, 8 \%$ and $10 \%$ SDS-PAGE gel and transferred to PDVF membrane (Millipore, USA). After blocking with 5\% non-fat milk for 1 hour at room temperature, membranes were incubated with primary antibodies specific 


\section{Cellular Physiology Cell Physiol Biochem 2018;49:190-205 \begin{tabular}{ll|l} 
DOI: 10.1159/000492853 & $\begin{array}{l}\text { O } 2018 \text { The Author(s). Published by S. Karger AG, Basel } \\
\text { www.karger.com/cpb }\end{array}$ \\
\hline and Biochemistry
\end{tabular}}

Feng et al:: Short-Chain Fatty Acids Protect Intestinal Barrier

for ZO-1 (1:1000, Invitrogen, CA), occludin (1:1000, Invitrogen), claudin-1 (1:1000, Invitrogen), claudin-2 (1:1000, Abcam, Germany), NLRP3(1:1000, Adipogen, USA), ASC (1:100, Santa Cruz, USA), caspase 1 (1:1000, Cell Signaling, USA), cleaved caspase 1(1:1000, Cell Signaling), IL-1 $\beta(1: 1000$, abcam, Germany), cleaved IL1ß(1:1000, Cell Signaling), IL-18(1:1000, abcam), LC3 (1:1000, Sigma), Atg5(1:1000, Sigma), p62(1:1000, Sigma), beclin $1\left(1: 1000\right.$, Sigma) and $\beta$-actin (1:5000, Sigma) overnight at $4^{\circ} \mathrm{C}$. After washing, membranes were incubated with peroxidase-conjugated secondary antibodies (1:5000, Southern Biotech, Birminghan, $\mathrm{AL}$ ) for 1 hour at room temperature. The blots were developed with an enhanced chemiluminescence kit (KeyGEN, China), and imaged using a ChemiDoc XRS system (Bio-Rad, CA). Densitometric analysis was performed using Quantity One software (Bio-Rad).

\section{Immunofluorescence microscopy}

Caco-2 monolayers grown on $0.33-\mathrm{cm}^{2}$ Transwell supports were washed with ice-cold PBS, fixed with $1 \%$ paraformaldehyde for $30 \mathrm{~min}$, incubated with $50 \mathrm{mmol} / \mathrm{L} \mathrm{NH}_{4} \mathrm{CL}$ for $15 \mathrm{~min}$, and permeabilized with $0.1 \%$ Triton X-100 in PBS containing $1 \mathrm{mmol} / \mathrm{L} \mathrm{CaCl}_{2}$ at room temperature. Then, monolayers were blocked in 2.5\% bovine serum albumin (BSA) and incubated with anti-ZO-1 (1:100, Invitrogen, CA), antioccludin (1:100, Invirtrogen), anti-ASC (1:50, Santa Cruze) or anti-LC3 (1:100, Sigma) antibodies diluted in PBS containing $2.5 \%$ BSA at $4{ }^{\circ}$ Covernight. Monolayers were washed with PBS followed by incubated with Alexa Fluor 488-conjugated anti-IgG secondary antibodies (1:100, EMD Chemicals, Gibbstown, NJ) and DAPI (Biotium Inc, Hayward, CA) for 1 hours at room temperature. After washing with PBS, monolayers were mounted in Slowfade (Molecular Probes) and imaged using a laser scanning fluorescence microscopy (TCS SP5, Leica, Germany).

\section{Determination of ROS production}

The intracellular ROS levels were detected with the employment of DCFH-DA, which was used to monitor intracellular-produced hydrogen peroxide $\left(\mathrm{H}_{2} \mathrm{O}_{2}\right)$ because it can be oxidised by intracellular oxidants and then emitted fluorescence [21]. To assay the effect of various reagents on ROS production, cells were seeded in plates for 3 days and then exposed to reagents. After 24 hours, 5umol/L DCFH-DA was added to the wells for $30 \mathrm{~min}$ at $37^{\circ} \mathrm{C}$, and cells were washed thrice with PBS quickly to remove the extra dye. The OD value of ROS was measured by a fluorescent microplate reader (Varioskan Flash, Thermo Electron Corporation, Vantaa, Finland) at excitation wavelength of $488 \mathrm{~nm}$ and emission wavelength of 525 $\mathrm{nm}$. The fluorescence intensity of ROS was measured by a fluorescence microscope (Lecia, Germany) with an excitation wavelength of $488 \mathrm{~nm}$ and an emission wavelength of $530 \mathrm{~nm}$.

\section{Statistical Analysis}

Results are shown as the mean \pm SEM. Differences among groups were assessed with One-way ANOVA by SPSS 13.0. $p<0.05$ was considered statistically significant. All reported significance levels reported twotailed $p$ values.

\section{Results}

SCFAs contribute to the formation of intestinal barrier in Caco-2 monolayers

It has been well demonstrated that SCFAs including butyrate are prominent energy resources of intestinal epithelial cell [22], therefore, to investigate the effect of SCFAs on the formation of intestinal barrier function in Caco-2 monolayers, we firstly detect the influence of each SCFA on intestinal barrier function using various concentration gradients after the Caco-2 cells were seeded on transwell supports for 24 hours. TER, an indicator of epithelial paracellular permeability to ions, was employed to evaluate barrier function. As shown in Fig. $1 \mathrm{~A}$, acetate $(0.5 \mathrm{mmol} / \mathrm{L})$, propionate $(0.01 \mathrm{mmol} / \mathrm{L})$ and butyrate $(0.01 \mathrm{mmol} / \mathrm{L})$ alone progressively and significantly increased TER over the time-course of the experiments starting from the first day, besides, a compound of the three SCFAs showed the synergistic effect in increasing TER. And the synergistic effect reached its peak when the Caco- 2 cells were incubated with the compound for 4 days. Secondly, we utilized immunoblotting to analyze the expression of Z0-1 and claudin-2. Z0-1 is a member of the membrane-associated 
Fig. 1. SCFAs contribute to the formation of intestinal barrier in Caco-2 monolayers. A. Caco-2 cells were treated with acetate, propionate and butyrate respectively or simultaneously one day after the cells were seeded on Transwell supports. Acetate $(0.5 \mathrm{mmol} / \mathrm{L})$ and butyrate $(0.01 \mathrm{mmol} / \mathrm{L})$, but not propionate $(0.01 \mathrm{mmol} / \mathrm{L})$ alone significantly increased TER. When added simultaneously, acetate, butyrate and propionate increased TER significantly. ${ }^{*} \mathrm{p}<0.05$ compared with control. $n=5$. B.The expression of ZO-1 increased, whereas claudin- 2 decreased. $\quad{ }^{*} \mathrm{p}<0.05, \quad{ }^{* *} \mathrm{p}<0.01$, ${ }^{* * *} \mathrm{p}<0.001$ compared with control. $\mathrm{n}=6$. C. Tight junction protein ZO-1 was stained by immunofluorescence.

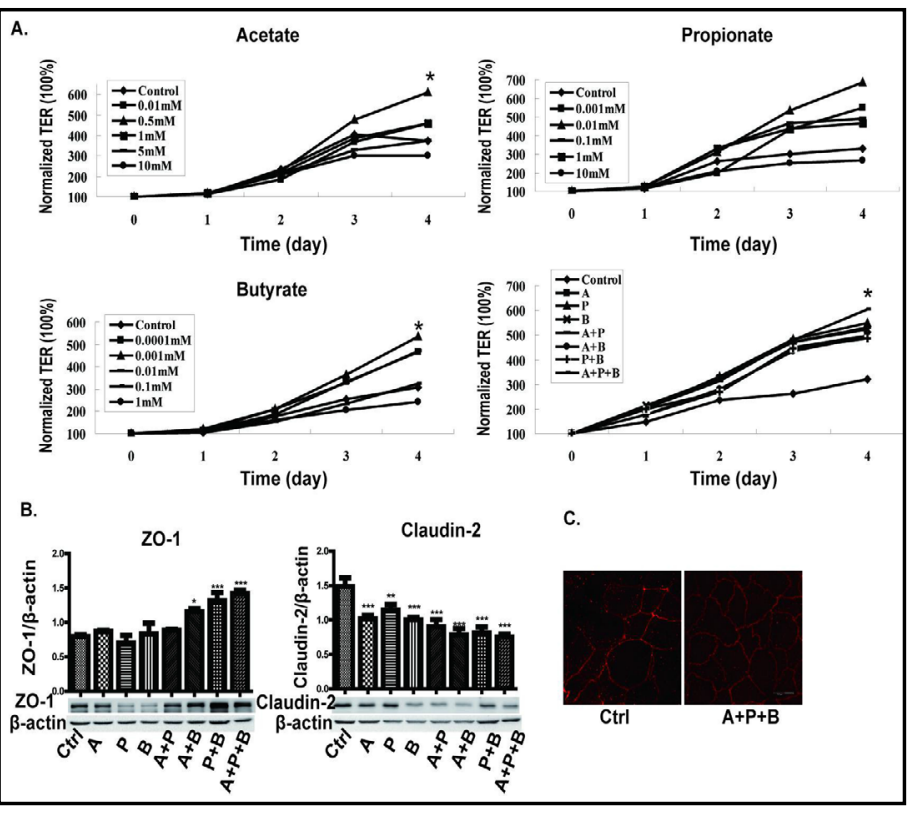
$\mathrm{n}=3$. Scale bar $=10 \mu \mathrm{m} . \mathrm{A}=$ acetate,

$\mathrm{P}=$ propionate, $\mathrm{B}=$ butyrate, $\mathrm{A}+\mathrm{P}=$ acetate + propionate, $\mathrm{A}+\mathrm{B}=$ acetate+butyrate, $\mathrm{P}+\mathrm{B}=$ propionate + butyrate, $\mathrm{A}+\mathrm{P}+\mathrm{B}=$ acetate + propionate+butyrate.

Fig. 2. SCFAs alleviate intestinal barrier dysfunction induced by LPS. A. Caco-2 monolayers were treated with LPS in the absence or presence of SCFAs for 24 hours. SCFAs significantly inhibited TER drop caused by LPS. ${ }^{* *} \mathrm{p}<0.001$ compared with control. \#\#\# $\mathrm{p}<0.001$ compared with LPS. $n=6$. B. The LPS-induced increase of paracellular permeability to $4 \mathrm{kD}$ FITC-dextran was lowered by SCFAs. ${ }^{*} \mathrm{p}<0.05$ compared with control. ${ }^{\#} \mathrm{p}<0.05$ compared with LPS. $n=5$. C. The protein expression was analyzed by Western blot. ${ }^{* *} \mathrm{p}<0.01,{ }^{* * *} \mathrm{p}<0.001$ compared with control. ${ }^{*} \mathrm{p}<0.05,{ }^{\# \#} \mathrm{p}<0.01$ compared with LPS. $n=6$. D. SCFAs alleviated the LPScaused morphological redistributions of $\mathrm{ZO}-1$ and occludin. $\mathrm{n}=3$. Scale bar $=10 \mu \mathrm{m}$. SCFAs here means a mixture of acetate $(0.5 \mathrm{mmol} / \mathrm{L})$, propionate $(0.01 \mathrm{mmol} / \mathrm{L})$ and butyrate $(0.01 \mathrm{mmol} / \mathrm{L})$, and the following are the same.
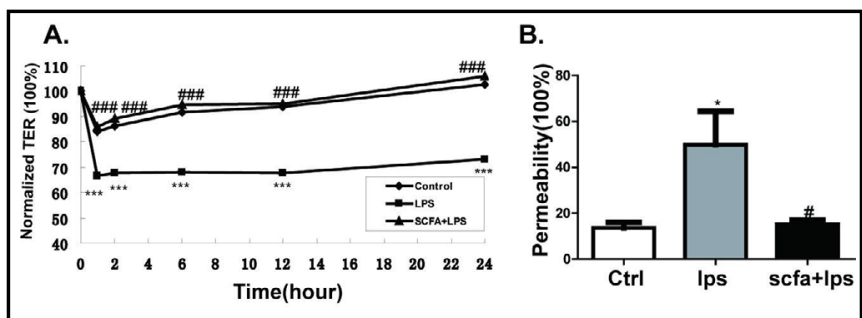

c.
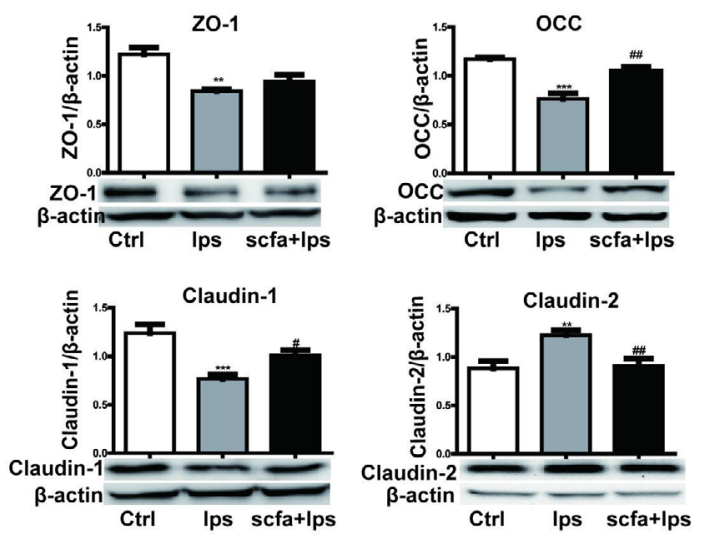

D.

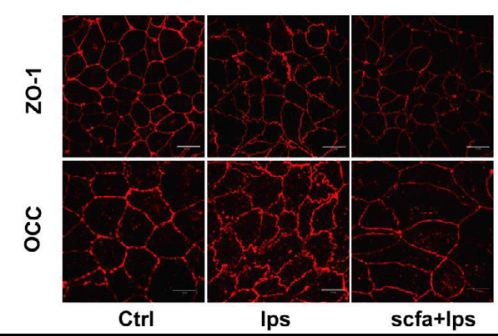




\section{Cellular Physiology

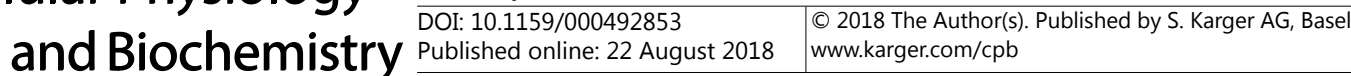 \\ Feng et al.: Short-Chain Fatty Acids Protect Intestinal Barrier}

guanylate kinase homologs and links to the transmembrane protein occludin [23]. Claudin-2 is a pore-forming protein [24]. As shown in Fig. $1 \mathrm{~B}$, all these three SCFAs alone decreased the expression of ZO-1 but increased the expression of claudin-2, and this was reversed when the cells were exposed to the compound for 4 days. Finally, we used immunofluorescence assay to analyze the distribution and morphology of ZO1. As illustrated in Fig. 1C, ZO-1 distributed along the cell membrane and looked like irregular undulating profiles after treatment with the compound for 4 days. By contrast, there was no ZO-1 existed in the control cells. These results indicate that the SCFAs possess the synergistic capacity to stimulate the formation of intestinal barrier in Caco-2 monolayers.

\section{SCFAs alleviate intestinal barrier dysfunction induced by LPS}

Previous studies have shown that SCFAs including butyrate enhance intestinal barrier function, and LPS disrupts intestinal barrier function $[7,8,25]$. In the present study, to investigate the effect of SCFAs on intestinal barrier dysfunction, the Caco-2 monolayers were treated with LPS $(10 \mu \mathrm{g} / \mathrm{ml})$ for 24 hours, without or with SCFAs. As shown in Fig. 2 A, the TER levels of control monolayers remained stable over the time-course of the experiment. However, LPS caused a remarkable decrease at 1 hour and maintained the tendency until 24 hours. By contrast, SCFAs significantly dampened the LPS-induced drop of TER. Besides, paracellular flux of FITC-dextran across Caco-2 monolayers, a measure of paracellular permeability, was also measured. As demonstrated in Fig. 2B, when

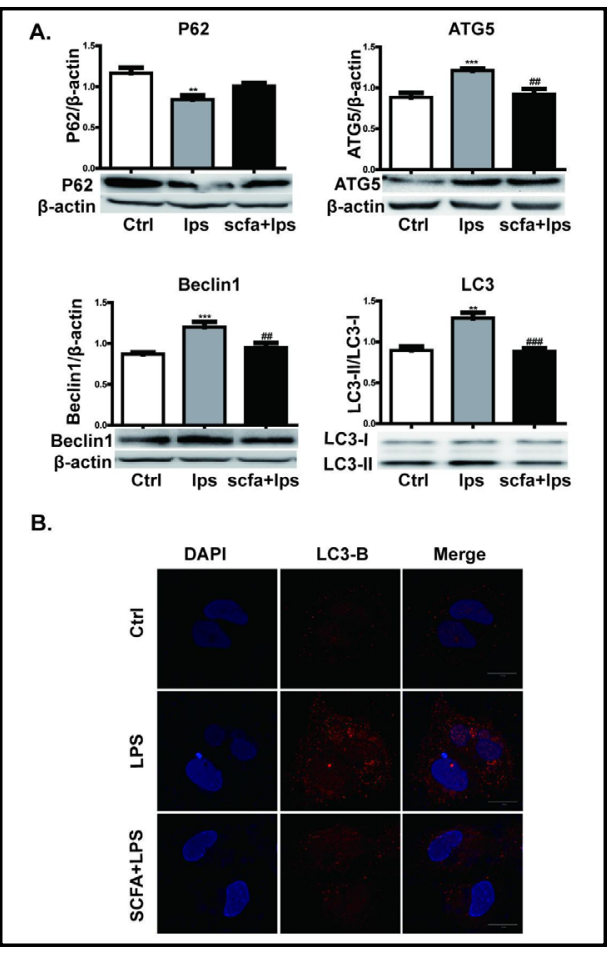

Fig. 3. SCFAs suppress the activation of autophagy induced by LPS. Caco-2 cells were treated with LPS in the absence or presence of SCFAs ro 24 hours. After the treatment of LPS for 24 hours, the protein expression of beclin-1, Atg5 and the ratio of LC3-II/ LC3-I increased, whereas p62 remarkably decreased. SCFAs statistically reversed the expression of these proteins, except p62. ${ }^{* *} \mathrm{p}<0.01,{ }^{* * *} \mathrm{p}<0.001$ compared with control. $\# \mathrm{p}<0.01$, \#\#\# $\mathrm{p}<0.001$ compared with LPS. $\mathrm{n}=6$. B. SCFAs remarkably inhibited the increase of the amount of LC3B caused by LPS. $n=3$. Scale bar $=10 \mu \mathrm{m}$. compared with the control, paracellular flux was significantly increased after treatment of LPS. In contrast, SCFAs dramatically diminished the LPS-induced increase of paracellular flux. Consistent with the changes of TER and paracellular flux, as presented in Fig. 2C, the expression of ZO-1, occludin and claudin-1 decreased, but claudin-2 increased in LPS group. By contrast, the LPS induced alteration of tight junction proteins was inhibited by SCFAs. At last, as shown in Fig. 2D, immunofluorescence assay showed that ZO-1 distribution transformed from regularly smooth arcs into irregularly undulating profiles and discontinuous punctate along the tight junction after the treatment of LPS for 24 hours. Similar to ZO-1, occludin distribution was also transformed into irregular arrangement, with partially transfer from the tight junction into cytoplasmic vesicles. However, SCFAs remarkably alleviated the morphological disturbances of ZO-1 and occludin. These results suggest that SCFAs protect intestinal barrier function from the disruption by LPS in Caco-2 monolayers. 


\section{Cellular Physiology Cell Physiol Biochem 2018;49:190-205 \begin{tabular}{ll|l} 
and Biochemistry & $\begin{array}{l}\text { DOI: 10.1159/000492853 } \\
\text { Published online: 22 August 2018 }\end{array}$ & $\begin{array}{l}\text { (c) } 2018 \text { The Author(s). Published by S. Karger AG, Basel } \\
\text { www.karger.com/cpb }\end{array}$
\end{tabular}

SCFAs suppress the activation of autophagy induced by LPS

It has been documented that autophagy can be induced by LPS and participates in the maintenance of intestinal homeostasis [26, 27], therefore, we further investigated autophagy in the present study. Microtubule-associated protein light chain (LC3), which is lipidated and incorporated into the autophagosomal membrane, is widely used as a marker for autophagy [15]. Besides, autophagy substrates include p62, Atg5 and beclin 1, among which p62 is an adaptor protein that binds to LC3, Atg 5 facilitates the phosphatidylethanolamine conjunction of LC3, and beclin 1 is a key regulator of the initiation of autophagy $[28,29,30]$. As shown in Fig. 3A, LPS induced a significant increase in the ratio of LC3-II (lipidated form) to LC3-I protein, Beclin 1 and Atg5, but a decrease in p62 expression. By contrast, SCFAs suppressed these alterations induced by LPS. Consistent with these findings, immunofluorescence assay showed a mass of red punctate substances, representing LC3B protein, in the cytoplasm of Caco-2 cells treated by LPS, whereas SCFAs reduced the amount of red punctate substances (Fig. 3B). These data confirm that SCFAs inhibit the activation of autophagy induced by LPS, and this might be one of the mechanisms by which SCFAs protect intestinal barrier function from the disruption by LPS in Caco- 2 cells.

SCFAs inhibit the activation of NLRP3 inflammasome induced by LPS

As NLRP3 infammasome can be activated by LPS through ROS production and regulates intestinal homeostasis [11,31], we further investigated NLRP3 inflammasome in Caco-2 cells treated with LPS for 24 hours. As indicated in Fig. 4A, LPS caused a remarkable elevation of NLRP3, ASC, caspase-1, cleaved-caspase-1, IL-1 $\beta$, cleaved-IL-1 $\beta$ and IL-18 in Caco-2 cells, however, SCFAs inhibited the LPS-induced elevation of these proteins. Consistent with these results, immunofluorescence assay showed that a mass of red specks representing ASC protein lie in cytoplasm of Caco-2 cells treated with LPS, however, SCFAs decreased the quantity of red spots (Fig. 4B). We further investigated the activation of reactive oxygen species (ROS). As indicated in Fig. 4C, both the OD value and fluorescence intensity were increased by LPS, and this was suppressed by SCFAs, indicating that SCFAs decrease the LPS-induced increase of ROS production. These findings confirm that SCFAs suppress NLRP3 inflammasome activated by LPS through the inhibition of ROS production in Caco- 2 cells, and this might be one possible mechanism by which SCFAs protect intestinal barrier disrupted by LPS.

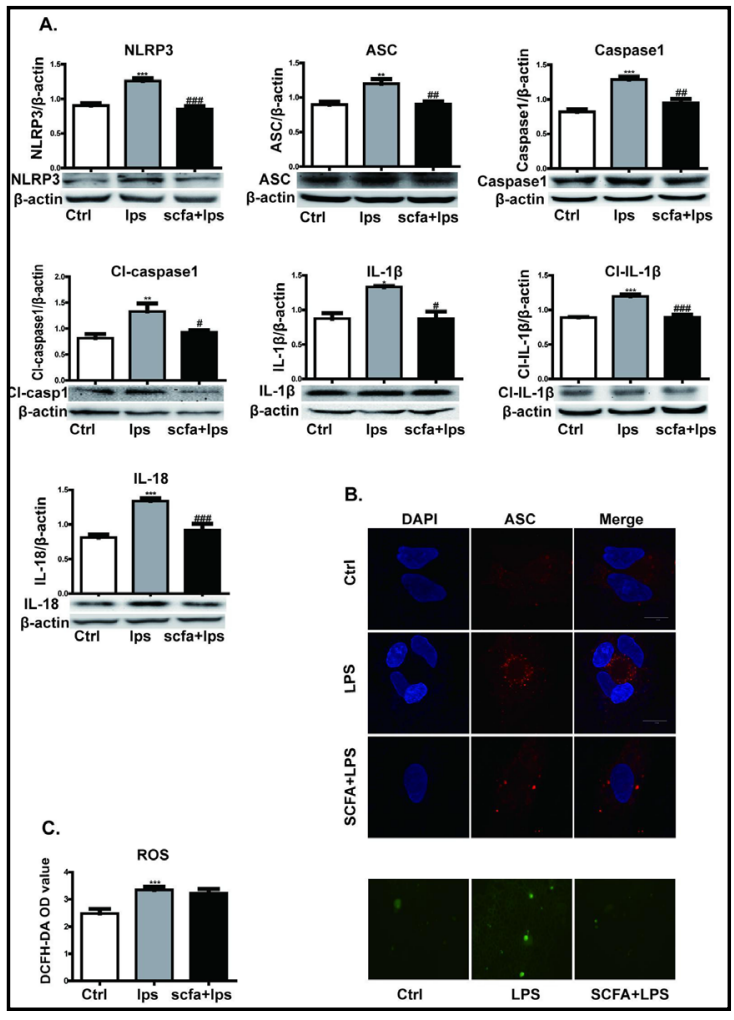

Fig. 4. SCFAs suppress the activation of NLRP3 inflammasome induced by LPS. A. Caco- 2 cells were treated with LPS in the absence or presence of SCFAs ro 24 hours. The protein expression of NLRP3, ASC, caspase- 1 , cleaved caspase- 1 , IL-1 $\beta$, cleaved IL-1 $\beta$ and IL-18 was increased by LPS, and SCFSs inhibited the LPS-caused increase of these proteins. ${ }^{*} \mathrm{p}<0.05$, $\mathrm{p}<0.01,{ }^{* * *} \mathrm{p}<0.001$ compared with control. ${ }^{*} \mathrm{p}<0.05$ remarkably inhibited the increase of the amount of ASC caused by LPS. Data are representative of three independent experiments. Scale bar $=10 \mu \mathrm{m}$. C. SCFAs significantly inhibited the increase of quantity and fluorescence intensity of ROS caused by LPS. ${ }^{* * *} \mathrm{p}<0.001$ compared with control. Scale bar $=25 \mu \mathrm{m} . \mathrm{n}=3$. 


\section{Cellular Physiology and Biochemistry Published \begin{tabular}{l|l} 
DOI: 10.1159/000492853 & $\begin{array}{l}\text { @ 2018 The Author(s). Published by S. Karger AG, Basel } \\
\text { www.karger.com/cpb }\end{array}$
\end{tabular} \\ Feng et al.: Short-Chain Fatty Acids Protect Intestinal Barrier}

Fig. 5. SCFAs act as HDAC inhibitors or energy substances. A1. Caco-2 monolayers were treated with trichostatin $\mathrm{A}$ and etomoxir respectively for 24 hours. Both trichostatin A and etomoxir significantly decreased TER. " $\mathrm{p}<0.05$, ${ }^{* * *} \mathrm{p}<0.001$ compared with control. $\mathrm{n}=5$. A2. Both trichostatin A and etomoxir remarkably decreased the paracellular permeability to $4 \mathrm{kD}$ FITCdextran. " $\mathrm{p}<0.05$ compared with control. $n=5$. A3. The protein expression of $\mathrm{ZO}-1$, occludin and claudin-1 statistically decreased by trichostatin A and etomoxir, however, etomoxir did not decrease claudin-2 statistically. ${ }^{*} \mathrm{p}<0.05,{ }^{* *} \mathrm{p}<0.01,{ }^{* * *} \mathrm{p}<0.001$ compared with control. $\mathrm{n}=6$. A4. $\mathrm{ZO}-1$ and occludin were stained by immunofluorescence. Both trichostatin A and etomoxir caused morphological disruption of ZO-1 and occludin. $n=3$. Scale bar= $10 \mu \mathrm{m}$. B1. Both trichostatin A and etomoxir significantly decreased the protein expression of p62, and increased the ratio of LC3-II/LC3-I. ${ }^{* * *} \mathrm{p}<0.001$ compared with control. $\mathrm{n}=6$. B2. Both trichostatin A and etomoxir remarkably increased the amount of LC3B. $n=3$. Scale bar $=10 \mu$ m. C1. Both trichostatin $\mathrm{A}$ and etomoxir statistically decreased the protein expression of NLRP3, ASC, caspase-1, cleaved caspase- 1 , cleaved IL-1 $\beta$ and IL-18, however, the decrease of IL-1 $\beta$ was without statistical significance. ${ }^{*} \mathrm{p}<0.05,{ }^{* *} \mathrm{p}<0.01,{ }^{* * *} \mathrm{p}<0.001$ compared with control. $\mathrm{n}=6$. C2. Both trichostatin

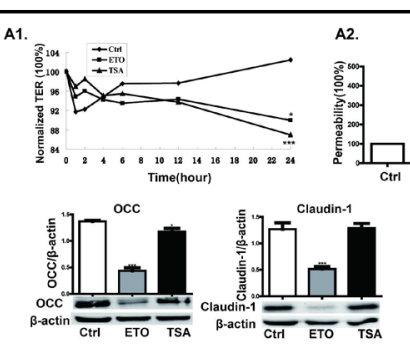

B1.
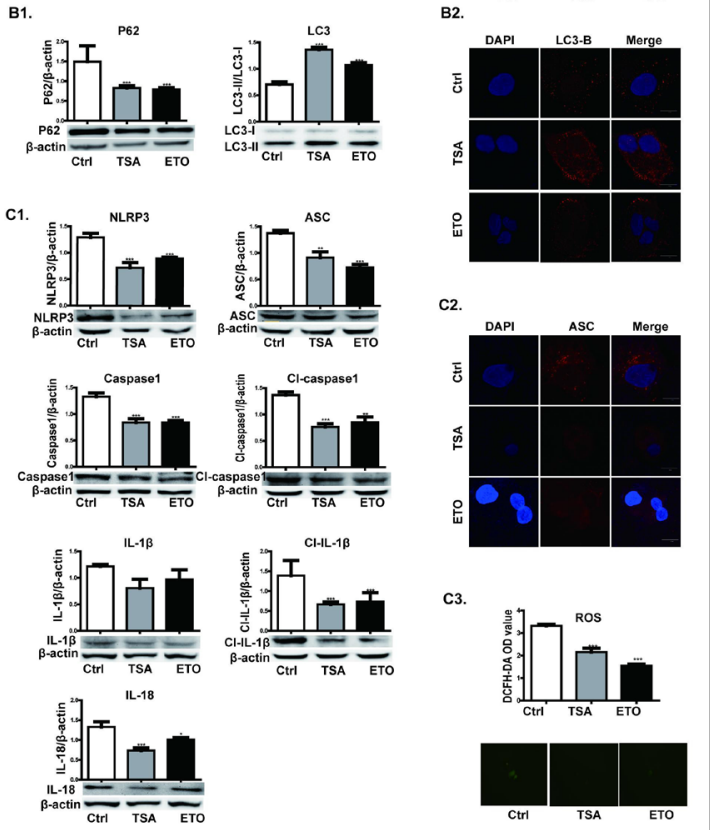

$A$ and etomoxir remarkably inhibited the amount

of red specks, representing ASC protein. $n=3$. Scale bar $=10 \mu \mathrm{m}$. C3. Both trichostatin A and etomoxir significantly inhibited the quantity and fluorescence intensity of ROS. ${ }^{* * *} \mathrm{p}<0.001$ compared with control. $\mathrm{n}=3$. Scale bar $=25 \mu \mathrm{m}$.

\section{SCFAs act as HDAC inhibitors or energy substances}

SCFAs may act as energy substances to protect intestinal barrier function. It is well illustrated that SCFAs may act as both the HDAC inhibitors and energy substances [6,9], therefore, we applied trichostatin A and etomoxir to identify whether SCFAs function as HDAC inhibitors or as energy substances to protect against LPS induced intestinal barrier dysfunction in Caco-2 cells. Trichostatin A and etomoxir respectively is a well-known HDAC inhibitor and a inhibitor of mitochondrial fatty acid $\beta$-oxidation [32, 33]. As shown in Fig. 5-A1, the TER levels progressively increased in control monolayers over the time-course, but significantly decreased in monolayers respectively treated with trichostatin A and etomoxir for 24 hours. Meanwhile, the paracellular flux of FITC-dextran across Caco-2 monolayers statistically increased (Fig. 5-A2). In addition, as indicated in Fig. 5-A3, both trichostatin A and etomoxir decreased the expressions of ZO-1, occludin and claudin-1. Furthermore, both trichostatin A and etomoxir destroyed the morphology of ZO-1 and occludin, as evidenced by that both proteins transformed from regularly smooth arcs into irregularly undulating profiles and discontinuous punctate along the tight junction(Fig. 5-A4). In terms of these changes, trichostatin A was more effective than etomoxir. Thus, SCFAs are more likely to function as energy substances rather than HDAC inhibitors to protect intestinal barrier function in Caco-2 cells. 
SCFAs may act as energy substances to suppress autophagy. It has been documented that energy metabolism is closely associated with autophagy in mammalian colon [17]. Thus, in the present study, we also investigated the effect of trichostatin A and etomoxir on autophagy activated by LPS in Caco-2 cells. As shown in Fig. 5-B1, trichostatin A and etomoxir significantly decreased protein expression of p62, however, increased the ratio of LC3-II (lipidated form) to LC3-I protein. Consistence with this, the quantity of red spots, which represent LC3B protein, also obviously increased, and trichostatin A showed a more prominent role (Fig. 5-B2). These indicate that SCFAs may function as energy substances to suppress autophagy activated by LPS in Caco-2 cells.

Fig. 6. NLRP3 inflammasome disrupts intestinal barrier function and activates autophagy. A1. Caco2 cells were treated with LPS $(1 \mu \mathrm{g} / \mathrm{ml})$ for 4 hours and then ATP $(5 \mathrm{mmol} / \mathrm{L})$ for $30 \mathrm{~min}$ with or without SCFAs. After the treatment, the protein expression of NLRP3, ASC, caspase-1, cleaved-caspase- 1 , IL-1 $\beta$, cleaved-IL-1 $\beta$ and IL-18 significantly increased, whereas SCFA remarkably suppressed the increase of these proteins. ${ }^{*} \mathrm{p}<0.05$, ${ }^{* *} \mathrm{p}<0.01, \quad{ }^{* * *} \mathrm{p}<0.001 \quad$ compared with control. ${ }^{\#} \mathrm{p}<0.05,{ }^{\# \#} \mathrm{p}<0.01$, \#\#\# $\mathrm{p}<0.001$ compared with NLRP3 group. $n=6$. A2. SCFAs statistically suppressed the amount of red specks increased by LPS and ATP. $n=3$. Scale bar $=10 \mu m$. A3. LPS and ATP significantly increased the fluorescence intensity of ROS, however, there was no statistical difference in the variation of the quantity of ROS production induced by NLRP3 induction. Scale bar $=25 \mu \mathrm{m} . \mathrm{n}=3$. B1. LPS and ATP significantly decreased TER, SCFAs remarkably reversed the decrease of TER. $\quad{ }^{* *}$ p $<0.01, \quad{ }^{* * *}$ p $<0.001$ compared with control. ${ }^{*} \mathrm{p}<0.01$ compared with NLRP3 group.

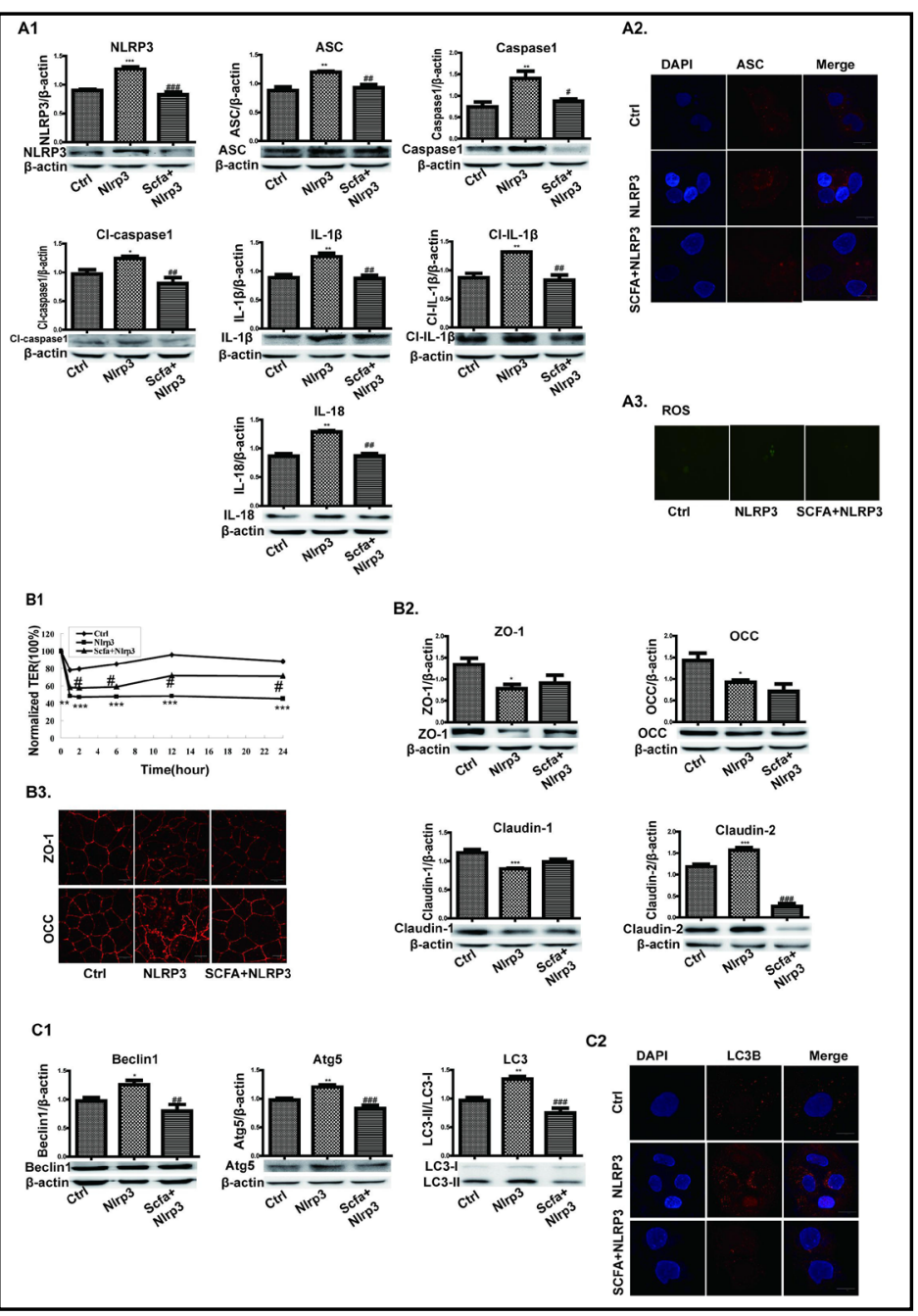
$n=4$. B2. The protein expression of

ZO-1, occludin and claudin-1 statistically decreased by LPS and ATP, however, the expression of claudin-2 significantly increased. SCFA reversed the variation tendency of ZO-1, occludin and claudin-1 without statistical significance, but notably inhibited the increase of claudin- 2 . ${ }^{*} \mathrm{p}<0.05,{ }^{* * *} \mathrm{p}<0.001$ compared

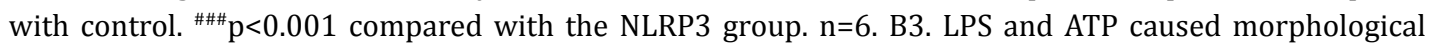
disruption of ZO-1 and occludin, and SCFA significantly prevented the morphological disruption of both ZO-1 and occludin. $\mathrm{n}=3$. Scale bar $=10 \mu \mathrm{m}$. C1. LPS and ATP significantly increased the ratio of LC3-II/LC3-I, and the expression of Beclin 1 and Atg5, and SCFA remarkably suppressed these variation tendencies. ${ }^{*} \mathrm{p}<0.05,{ }^{* *} \mathrm{p}<0.01$ compared with control. ${ }^{\# \#} \mathrm{p}<0.01$, ${ }^{\# \# \#} \mathrm{p}<0.001$ compared with NLRP3 group. $\mathrm{n}=6$. C2. LPS and ATP significantly increased the amount of red specks, representing LC3B proteins, which was inhibited by SCFAs. $n=3$. Scale bar $=10 \mu \mathrm{m}$. NLRP3 group means the group treated by LPS and ATP simultaneously.

\section{KARGER}


SCFAs may act as HDAC inhibitors to suppress NLRP3 inflammasome. The HDAC inhibitors have been reported to suppress inflammation [34]. We next determined the effect of trichostatin A and etomoxir on NLRP3 inflammasome activated by LPS in Caco2 cells. As shown in Fig. 5-C1, trichostatin A and etomoxir reduced the protein expression of NLRP3, ASC, caspase-1, cleaved-caspase-1, IL-1 $\beta$, cleaved-IL-1 $\beta$ and IL-18. Similarly, immunofluorescence assay showed that the amount of red specks was decreased notably (Fig. 5-C2). Furthermore, as indicated in Fig. 5-C3, the OD value and fluorescence intensity of ROS were decreased by both trichostatin A and etomoxir in Caco-2 cells treated with LPS for 24 hours. These results suggest that SCFAs may act as HDAC inhibitors to suppress NLRP3 inflammasome activated by LPS through the inhibition of ROS production in Caco- 2 cells.

Fig. 7. Autophagy destroys intestinal barrier function and activates NLRP3 inflammasome. A1. Caco-2 cells were treated with rapamycin (100 $\mathrm{nmol} / \mathrm{L})$ for 24 hours with or without SCFA. Rapamycin significantly increased the ratio of LC3-II/ LC3-I and the expression of Beclin 1 and Atg5, and remarkably decreased the expression of p62. SCFAs remarkably reversed the rapamycin-induced changes of these proteins. ${ }^{*} \mathrm{p}<0.05,{ }^{* *} \mathrm{p}<0.01$, ${ }^{* * *} \mathrm{p}<0.05$ compared with control. \# $\mathrm{p}<0.01 \quad \# \mathrm{p}<0.01, \quad \# \# \mathrm{p}<0.001$ compared with rapamycin. $\mathrm{n}=6$. A2. Rapamycin significantly increased the amount of red specks, representing LC3B proteins, which was inhibited by SCFAs. $n=3$. Scale bar $=10 \mu \mathrm{m}$. B1. Rapamycin significantly decreased TER, and SCFA remarkably reversed the decrease of TER. ${ }^{*} p<0.05,{ }^{* *} \mathrm{p}<0.01$ compared with control. ${ }^{\# \#} \mathrm{p}<0.01$, $\# \#$ p $<0.001$ compared with rapamycin. $n=5$ or 6 . $B 2$. The protein expression of ZO-1, occludin and claudin-1 were statistically decreased by rapamycin, however, the expression of claudin- 2 was increased. SCFA reversed the rapamycin-induced changes of these proteins. ${ }^{*} p<0.05,{ }^{* *} p<0.05$,

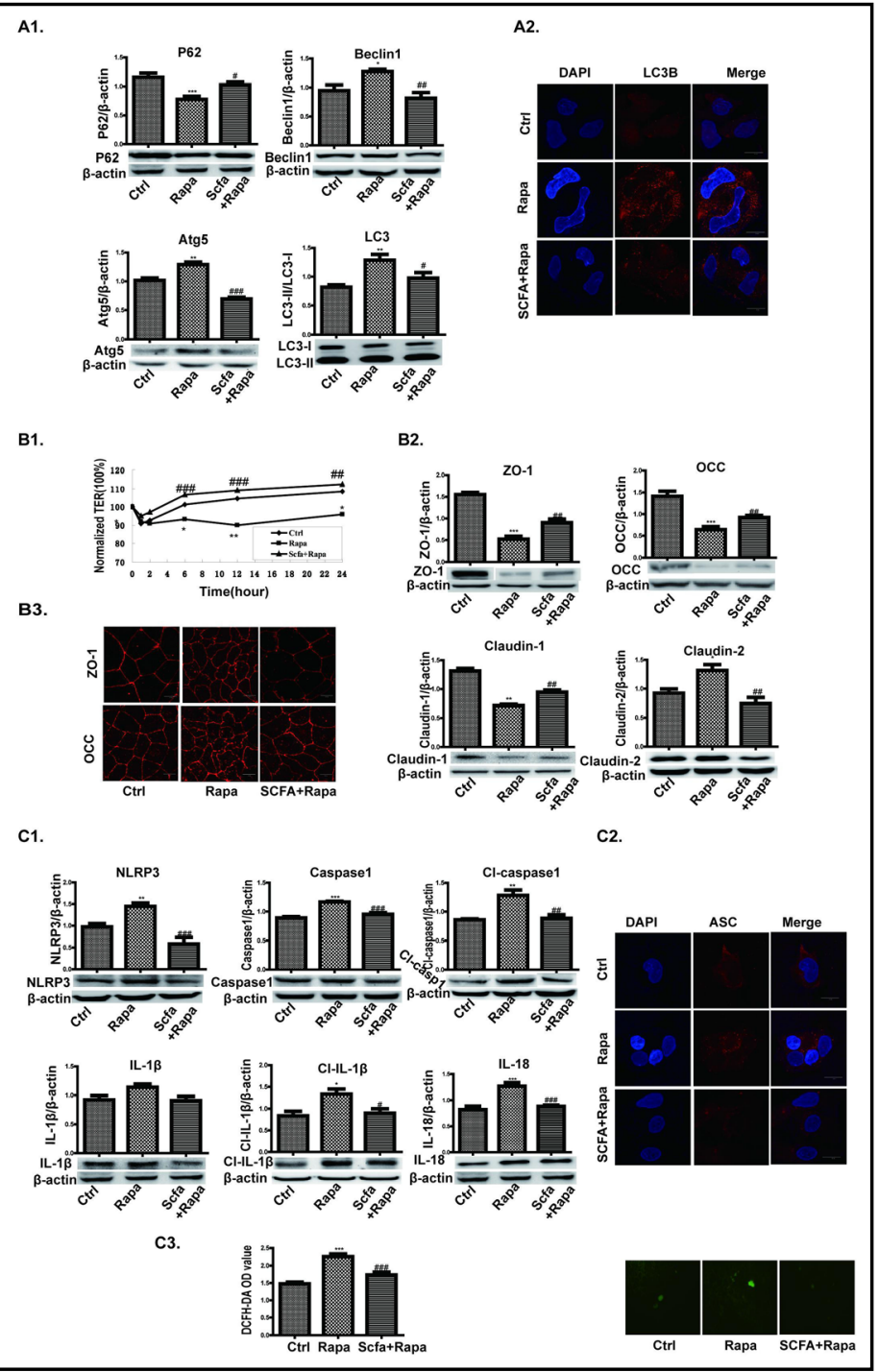
${ }^{* * *} \mathrm{p}<0.001$ compared with control.

\#\# $\mathrm{p}<0.01$ compared with the rapamycin. $\mathrm{n}=6$. B3. Rapamycin caused morphological disruption of Z0-1 and occludin, which was significantly prevented by SCFAs. $n=3$. Scale bar $=10 \mu \mathrm{m}$. C1. Rapamycin significantly increased the protein expression of NLRP3, ASC, caspase-1, cleaved-caspase-1, cleaved-IL-1 $\beta$ and IL-18, which was remarkably suppressed by SCFAs. ${ }^{*} \mathrm{p}<0.05,{ }^{* *} \mathrm{p}<0.01,{ }^{* * *} \mathrm{p}<0.001$ compared with control. ${ }^{*} \mathrm{p}<0.05$, $\#$ p $<0.01, \# \# p<0.001$ compared with rapamycin. $n=6$. C2. SCFA statistically suppressed the amount of red specks increased by rapamycin. $n=3$. Scale bar $=10 \mu \mathrm{m}$. C3. Rapamycin significantly increased the quantity and fluorescence intensity of ROS, which was notably suppressed by SCFAs. Scale bar= $25 \mu \mathrm{m} . \mathrm{n}=3$. 


\section{Cellular Physiology Cell Physiol Biochem 2018;49:190-205 \begin{tabular}{l|l|l} 
and Biochemistry Published online: 22 August 2018 & $\begin{array}{l}\text { (c) } 2018 \text { The Author(s). Published by S. Karger AG, Basel } \\
\text { www.karger.com/cpb }\end{array}$
\end{tabular}}

NLRP3 inflammasome disrupts intestinal barrier function and activates autophagy NLRP3 inflammasome could be activated by LPS and ATP. It is well documented that NLRP3 inflammasome is activated by simultaneous incubation with LPS and ATP at appropriate concentrations [14]. In the present study, Caco-2 cells were exposed to LPS $(1 \mu \mathrm{g} / \mathrm{ml})$ for 4 hours and then ATP ( $5 \mathrm{mmol} / \mathrm{L})$ for $30 \mathrm{~min}$ to induce the activation of NLRP3 inflammasome. As shown in Fig. 6-A1, the protein expression of NLRP3, ASC, caspase-1, cleaved-caspase-1, IL-1 $\beta$, cleaved-IL-1 $\beta$ and IL-18 elevated significantly after the induction treatment, and the elevated expression of these proteins was inhibited by SCFAs. Similarly, as illustrated in Fig. 6-A2, immunofluorescence assay showed that the amount of red specks, representing ASC protein, increased in the cytoplasm of Caco- 2 cells after the induction treatment, whereas SCFAs reduced the amount of red specks. Furthermore, as shown in Fig. 6-A3, fluorescence intensity of ROS increased after the induction treatment, which was also alleviated by SCFAs. These results imply that NLRP3 inflammasome is activated by LPS and ATP, which is suppressed by SCFAs through ROS inhibition.

NLRP3 inflammasome destroyed intestinal barrier function. To further confirm the effect of NLRP3 inflammasome on intestinal barrier function, we analyzed intestinal barrier function after the NLRP3 inflammasome was activated by LPS and ATP. As shown in Fig. 6-B1, TER was significant reduced after the NLRP3 inflammasome was activated, whereas SCFAs alleviated the decrease of TER. Similarly, as indicated in Fig. 6-B2, activation of NLRP3 inflammasome notably decreased the proteins expressions of ZO-1, occludin and claudin-1, and increased claudin-2 expression, whereas SCFAs significantly alleviated the situation. Furthermore, immunofluorescence assay showed that the morphology of ZO-1 and occludin was seriously destroyed, with the transformation of both proteins from regularly smooth arcs into irregularly undulating profiles and discontinuous punctate along the tight junction (Fig. 6-B3). By contrast, SCFAs significantly alleviated this destruction induced by NLRP3 inflammasome activation. Taken together, these findings suggest that intestinal barrier function is destroyed by NLRP3 inflammasome activation, which can be alleviated by SCFAs in Caco-2 monolayers.

NLRP3 inflammasome induces the activation of autophagy. Based on previous findings [35], we were interested in whether the activation of NLRP3 inflammasome could induce autophagy. With this purpose, we further studied autophagy in Caco- 2 cells after the NLRP3 inflammasome was activated. As shown in Fig. 6-C1, after the activation of NLRP3 inflammasome, the ratio of LC3-II to LC3-I, and the protein expression of Beclin 1 and Atg5 all increased significantly, whereas SCFAs reversed the increase. Similarly, as indicated in Fig. 6-C2, the amount of red specks, representing LC3B, remarkably elevated after NLRP3 inflammasome was activated, but SCFAs also reduced the amount of red specks. These data indicate that NLRP inflammasome induces the activation of autophagy, which SCFAs could be suppressed by SCFAs.

Autophagy destroys intestinal barrier function and activates NLRP3 inflammasome

Autophagy is activated by rapamycin. It is well known that rapamycin acts as a activator of autophagy [36], therefore, we employed rapamycin (100 nmol/L) to induce autophagy. As shown in Fig. 7-A1, treatment with rapamycin for 24 hours significantly increased the ratio of LC3II to LC3-I and the expression of Beclin 1 and Atg5, and decreased p62 expression, which was remarkably alleviated by SCFAs. Furthermore, as indicated in Fig. 7-A2, immunofluorescence assay show that the amount of red specks, representing LC3B protein, increased significantly after rapamycin treatment. By contrast, SCFAs reduced the amount of red specks. These suggest that autophagy is activated by rapamycin, and SCFAs could suppress the activation of autophagy in Caco-2 cells.

Autophagy disrupts intestinal barrier function. To further investigate the effect of autophagy on intestinal barrier function, we assessed intestinal barrier function after the autophagy was activated by rapamycin. As shown in Fig. 7-B1, TER significant reduced after the autophagy was activated, whereas SCFAs remarkably reversed the drop of TER. In 


\section{Cellular Physiology Cell Physiol Biochem 2018:49:190-205 \begin{tabular}{l|l|l} 
and Biochemistry 10.1159/000492853 & $\begin{array}{l}\text { (C) 2018 The Author(s). Published by S. Karger AG, Basel } \\
\text { www.karger.com/cpb }\end{array}$
\end{tabular} \\ Feng et al.: Short-Chain Fatty Acids Protect Intestinal Barrier}

addition, as indicated in Fig. 7-B2, the expression of ZO-1, occludin and claudin-1 decreased significantly, and claudin-2 expression increased significantly after the autophagy was activated. Similarly, SCFAs notably reversed the alterations of these proteins. Furthermore, as illustrated in Fig. 7-B3, both of ZO-1 and occludin transformed from regularly smooth arcs into irregularly undulating profiles and discontinuous punctate along the tight junction, and SCFAs ameliorated the destructed morphology of ZO-1 and occludin. These data suggest that activating autophagy leads to the intestinal barrier dysfunction, which could be ameliorated by SCFAs in Caco-2 cells.

Autophagy activates NLRP3 inflammasome. Based on our results, we further determined whether the activation of autophagy could activate NLRP3 inflammasome. As shown in Fig. $7-\mathrm{C} 1$, the proteins expressions of NLRP3, caspase- 1 , cleaved-caspase- 1 , IL-1 $\beta$, cleaved-IL$1 \beta$ and IL-18 increased significantly after autophagy was activated, and SCFAs inhibited the elevations of these proteins. Furthermore, as indicated in the Fig. 7-C2, the amount of red specks, representing ASC protein, increased notably after the activation of autophagy, whereas SCFAs diminished the amount of red specks. Meanwhile, both the fluorescence intensity and OD value increased after autophagy was activated, but was suppressed by SCFAs, as illustrated in Fig. 7-C3. Taken together, these results indicate that NLRP3 inflammasome can be activated by autophagy, which is suppressed by SCFAs through inhibiting ROS production.

\section{Discussion}

The intestinal barrier plays an important role in maintaining intestinal homeostasis. Defects in the epithelial barrier contribute to the development of intestinal inflammation by allowing host immune system access to luminal antigens and micobes [19]. Emerging evidences show that SCFAs can repair the dysfunction of intestinal barrier and function as energy substances or HDAC inhibitors [7, 8, 9, 33]. In addition, NLRP3 inflammasome and autophagy participate in the maintenance of intestinal barrier function. In particular, it has been well documented that NLRP3 inflammasome and autophagy are respectively involved in the pathogenesis of DSS-induced colitis and Crohn disease [19, 37]. However, the molecular mechanisms of SCFAs on intestinal barrier function are still unclear. In the present study, we investigated the stimulative and protective effect of SCFAs on intestinal barrier function, and the underlying mechanisms. Our data show that SCFAs stimulate the formation of intestinal barrier function, and protect intestinal barrier dysfunction induced by LPS through inhibiting the activation of NLRP3 inflammasome and autophagy. SCFAs act as energy substances to protect intestinal barrier and inhibit autophagy but act as HDAC inhibitors to suppress NLRP3 inflammasome. Fourth, NLRP3 inflammasome and autophagy destroys intestinal barrier function after they were activated by LPS+ATP and rapamycin respectively. Fifth, both NLRP3 inflammasome and autophagy manifest interactive function with each other.

SCFAs, including acetate, propionate and butyrate, are major byproducts of dietary fiber in intestine during fermentation [6]. Previously in vitro and in vivo studies have shown that SCFAs protect the intestinal barrier integrity $[7,8,38]$. For example, sodium butyrate was reported to protect and strengthen epithelial barrier function in cdx2-IEC monolayers by up-regulating claudin-1 transcription, both in physiological and pathological conditions [7]. In a rat model of ischemia and reperfusion (I/R), butyrate was proved to act as a potential strategy to prevent intestinal I/R injury [38]. In agreement with these previous studies, our present study reveals that SCFAs simulate the formation of intestinal barrier in physiological condition. SCFAs also protect the intestinal barrier disruption induced by LPS.

The mechanisms by which SCFAs protect intestinal barrier dysfunction are complex and not fully clarified. Previous studies have demonstrated that SCFAs are involved in the protection of intestinal barrier function by increasing claudin-1 transcription [7], interacting with intracellular energy sensor AMPK[8], suppressing inflammation [6, 39]. The activation 
of autophagy and NLRP3 inflammasome has been reported to play a critical role in the regulation of epithelial barrier function $[14,19]$. In this study, we show that SCFAs inhibit the activation of autophagy and NLRP3 inflammasome induced by LPS. In consistent with our finding, butyrate was reported to inhibit autophagy in colonocytes [17]. However, butyrate was shown to promote NLRP3 inflammasome in vitro [40]. Based on our results, it is suggested that the mechanism by which SCFAs protect intestinal barrier dysfunction induced by LPS may, at least in part, be attributed to the inhibition of autophagy and NLRP3 inflammasome.

It has been previously described that inhibiting the $\beta$-oxidation of SCFAs decreased its function of recovering intestinal permeability [41]. HDAC such as HDAC 3 was reported to play a central role in maintaining intestinal homeostasis [42]. SCFAs was documented to act as HDAC inhibitors and energy substances $[6,9]$. Thus, in present study we investigated the effects of trichostatin A, the HDAC inhibitor, and etomoxir, the $\beta$-oxidation inhibitor, on intestinal barrier, autophagy and NLRP3 inflammasome. Our results demonstrate that SCFAs act as energy substances to protect intestinal barrier function and inhibit autophagy. These results are consistent with the previous studies showing that butyrate acts as an energy source to inhibit autophagy [17], and interacts with intracellular energy sensor AMPK to enhance intestinal barrier [8]. Besides, we also show that SCFAs act as HDAC inhibitors to suppress NLRP3 inflammasome. In contrary to our finding, HBI-800, another HDAC inhibitor, was reported to activate NLRP3 inflammasome in adult T-cell leukemia/lymphoma [43]. This discrepancy may be attributed to the different cell type, the different HDAC inhibitor, or other unknown reasons, which needs to be clarified further.

It has been reported that NLRP3 inflammasome plays an important role in the maintenance of gut homeostasis [40], and that autophagy deficiency in macrophages enhances NLRP3 inflammasome activity [44]. Thus, to investigate the effect of activated NLRP3 inflammasome on intestinal epithelial barrier, Caco-2 cells were treated with LPS and ATP to induce the activation of NLRP3 inflammasome, as previously described [14]. The treatment with LPS and ATP resulted in increased expression of NLRP3, ASC, caspase-1, cleaved-caspase-1, IL-1 $\beta$, cleaved-IL-1 $\beta$ and IL-18, implying the NLRP3 inflammasome was certainly activated in Caco-2 cells. More importantly, the LPS and ATP-induced activation of NLRP3 inflammasome remarkably caused the barrier disruption in Caco- 2 monolayers, as evidenced by the reductions of TER and expression of ZO-1, occludin and claudin-1, the increase of claudin-2 expression, and the relocalization of both ZO-1 and occludin. These findings are in line with previous report showing that the activated NLRP3 inflammasome led to the disruption of epithelial barrier function by down-regulating the expressions of ZO-1 and E-cadherin in cholangiocytes [14]. Meanwhile, our present data also revealed that the activation of NLRP3 inflammasome triggered by LPS and ATP induced the activation of autophagy in Caco-2 cells, which is consistent with the previous study showing that NLRP3 inflammasome activation could induce mitophagy/autophagy in peritoneal mesothelial cells [45]. On the contrary, several evidences illustrate that inhibiting NLRP3 inflammasome would trigger autophagy activation [46]. Hence, it is necessary to identify the precise relationship between NLRP3 inflammasome activation and autophagy activation.

Autophagy has intimate relationship with intestinal epithelial tight junction barrier $[15,19,28]$, and could activate NLRP3 inflammasome [47]. Therefore, to determine the role of autophagy activation in regulating intestinal epithelial barrier, Caco- 2 cells were treated with rapamycin, a well-known autophagy activator, to induce autophagy. The treatment with rapamycin increased the expression of Atg5 and Beclin 1 and the ratio of LC3-II/LC3-I, and decreased p62 expression, implying that autophagy was indeed activated in Caco-2 cells treated with rapamycin. Remarkably, the activation of autophagy could impair the barrier function of Caco-2 monolayers through down-regulating the expression of ZO-1, occludin and claudin-1, up-regulating claudin-2 expression, and destroying the morphology of both ZO-1 and occludin. Similarly, inhibition of autophagic degradation process was reported to contribute to epithelial tight junction dysfunction in TNF- $\alpha$ treated cell monolayers [48]. However, autophagy was demonstrated to enhance tight junction through down-

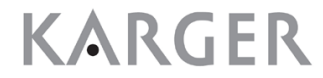




\section{Cellular Physiology Cell Physiol Biochem 2018;49:190-205 \begin{tabular}{ll|l} 
DOI: 10.1159/000492853 & $\begin{array}{l}\text { O 2018 The Author(s). Published by S. Karger AG, Basel } \\
\text { www.karger.com/cpb }\end{array}$ \\
\hline
\end{tabular} \\ Feng et al.: Short-Chain Fatty Acids Protect Intestinal Barrier}

regulating claudin-2 expression [19]. It is worth noting that enhancing autophagy induced the activation of NLRP3 inflammasome in Caco-2 cells. In contrast, it was reported that inducing autophagy suppressed the activation of NLRP3 inflammasome in rat [47], and that autophagy inhibition contributed to NLRP3 inflammasome activation in macrophages [49]. Therefore, the exact relationship between autophagy and NLRP3 inflammasome is still a complex and controversial issue to be clarified.

In conclusion, our findings demonstrate that SCFAs contribute to the formation of intestinal barrier at proper concentration, and possess the capability to ameliorate the LPSinduced intestinal barrier disruption by suppressing NLRP3 inflammasome and autophagy via inhibition of ROS production. SCFAs act as energy substances to influence intestinal barrier and autophagy, but act as HDAC inhibitor to affect NLRP3 inflammasome. Furthermore, the mutual promoting action between NLRP3 inflammsome and autophagy would lead to the intestinal barrier dysfunction, which could be alleviated by SCFAs. These findings provide a more detailed understanding of the role of SCFAs, even if there are some unresolved issues such as the precise relationship between autophagy and NLRP3 inflammasome.

\section{Acknowledgements}

This work was supported by the National Natural Science Foundation of China (81772081), and the program for Changjiang Scholars and Innovative Team (IRT13050).

Fengjun Wang designed the experiments. Yanhai Feng, Yalan Huang, Yu Wang and Pei Wang made substantial contributions to acquisition, analysis and interpretation of data. Yanhai Feng and Fengjun Wang wrote the manuscript.

\section{Disclosure Statement}

The authors declare that the research was conducted in the absence of any commercial or financial relationships that could be construed as a potential conflict of interests.

\section{References}

1 Marchiando AM, Graham WV, Turner JR: Epithelial Barriers in Homeostasis and Disease. Ann Rev Pathol 2010;5:119-144.

-2 Turner JR: Intestinal mucosal barrier function in health and disease. Nat Rev Immunol 2009;9:799-809.

- 3 Shen L, Weber CR, Raleigh DR, Yu D, Turner JR: Tight Junction Pore and Leak Pathways: A Dynamic Duo. Annu Rev Physiol 2011;73:283-309.

4 Salim SY, Söderholm JD: Importance of Disrupted Intestinal Barrier in Inflammatory Bowel Diseases. Inflamm Bowel Dis 2011;1:362-381.

-5 McGuckin MA, Eri R, Simms LA, Florin TH, Radford-Smith G: Intestinal barrier dysfunction in inflammatory bowel diseases. Inflamm Bowel Dis 2009;15:100-113.

-6 Cushing K, Alvarado DM, Ciorba MA: Butyrate and Mucosal Inflammation: New Scientific Evidence Supports Clinical Observation. Clin Transl Gastroenterol 2015;6:e108.

-7 Wang HB, Wang PY, Wang X, Wan YL, Liu YC: Butyrate Enhances Intestinal Epithelial Barrier Function via Up-Regulation of Tight Junction Protein Claudin-1 Transcription. Dig Dis Sci 2012;57:3126-3135.

8 Peng L, Li ZR, Green RS, Holzman IR, Lin J: Butyrate Enhances the Intestinal Barrier by Facilitating Tight Junction Assembly via Activation of AMP-Activated Protein Kinase in Caco-2 Cell Monolayers. J Nutr 2009;139:1619-1625.

-9 Jin UH, Cheng Y, Park H, Davidson LA, Callaway ES, Chapkin RS, Jayaraman A, Asante A, Allred C, Weaver EA, Safe S: Short Chain Fatty Acids Enhance Aryl Hydrocarbon (Ah) Responsiveness in Mouse Colonocytes and Caco-2 Human Colon Cancer Cells. Sci Rep 2017;7:10163. 


\section{Cellular Physiology Cell Physiol Biochem 2018;49:190-205 \begin{tabular}{l|l|l} 
and Biochemistry Published online: 22 August 2018 & $\begin{array}{l}\text { @ } 2018 \text { The Author(s). Published by S. Karger AG, Basel } \\
\text { www.karger.com/cpb }\end{array}$ \\
\hline
\end{tabular}}

10 Zhang Q, Yang F, Li X, Zhang HY, Chu XG, Zhang H, Wang LW, Gong ZJ: Trichostatin A protects against intestinal injury in rats with acute liver failure. J Surg Res 2016;205:1-10.

11 Hirota SA, Ng J, Lueng A, Khajah M, Parhar K, Li Y, Lam V, Potentier MS, Nq K, Bawa M, McCafferty DM, Rioux KP, Ghosh S, Xavier RJ, Colgan SP, Tschopp J, Muruve D, MacDonald JA, Beck PL: NLRP3 inflammasome plays a key role in the regulation of intestinal homeostasis. Inflamm Bowel Dis 2011;17:1359-1372.

12 Petrilli V, Dostert C, Muruve DA, Tschopp J: The inflammasome: a danger sensing complex triggering innate immunity. Curr Opin Immunol 2007;19:615-622.

13 Yu X, Lan P, Hou X, Han Q, Lu N, Li T, Jiao C, Zhang J, Zhang C, Tian Z: HBV inhibits LPS-induced NLRP3 inflammasome activation and IL-1b production via suppressing the NF-jB pathway and ROS production. J Hepatol 2017;66:693-702.

14 Maroni L, Agostinelli L, Saccomanno S, Pinto C, Giordano DM, Rychlicki C, De Minicis S, Trozzi L, Banales JM, Melum E, Karlsen TH, Benedetti A, Baroni GS, Marzioni M: Nlrp3 Activation Induces Il-18 Synthesis and Affects the Epithelial Barrier Function in Reactive Cholangiocytes. Am J Pathol 2017;187:366-376.

15 Levine B, Kroemer G: Autophagy in the Pathogenesis of Disease. Cell 2008;132:27-42.

16 Fan C, Liu Y, Zhao M, Zhan R, Cui W, Jin H, Teng Y, Lv P, Zheng L, Huang Y: Autophagy Inhibits C2-ceramidemediated Cell Death by Decreasing the Reactive Oxygen Species Levels in SH-SY5Y Cells. Neurosci Lett 2017;651:198-206.

17 Donohoe DR, Garge N, Zhang X, Sun W, O'Connell TM, Bunger MK, Bultman SJ: The Microbiome and Butyrate Regulate Energy Metabolism and Autophagy in the Mammalian Colon. Cell Metab 2011;13:517526.

18 Sakiyama T, Musch MW, Ropeleski MJ, Tsubouchi H, Chang EB: Glutamine increases autophagy under basal and stressed conditions in intestinal epithelial cells. Gastroenterology 2009;136:924-932.

19 Nighot PK, Hu CA, Ma TY: Autophagy Enhances Intestinal Epithelial Tight Junction Barrier Function by Targeting Claudin-2 Protein Degradation. J Biol Chem 2015;290:7234-7246.

-20 Wang F, Graham WV, Wang Y, Witkowski ED, Schwarz BT, Turner JR: Interferon- $\gamma$ and Tumor Necrosis Factor- $\alpha$ Synergize to Induce Intestinal Epithelial Barrier Dysfunction by Up-Regulating Myosin Light Chain Kinase Expression. Am J Pathol 2005;2:409-419.

-21 Guo X, Jiang X, Ren X, Sun H, Zhang D, Zhang Q Zhang J, Huang Y: The Galvanotactic Migration of Keratinocytes is Enhanced by Hypoxic Preconditioning. Sci Rep 2015;5:10289.

22 Cushing K, Alvarado DM, Ciorba MA: Butyrate and Mucosal Inflammation: New Scientific Evidence Supports Clinical Observation. Clin Transl Gastroenterol 2015;6:e108.

23 Fanning AS, Jameson BJ, Jesaitis LA, Anderson JM: The Tight Junction Protein ZO-1 Establishes a Link between the Transmembrane Protein Occludin and the Actin Cytoskeleton. J Biol Chem 1998;273:2974529753.

24 Krause G, Winkler L, Mueller SL, Haseloff RF, Piontek J, Blasiq IE: Structure and function of claudins. Biochim Biophys Acta 2008;1778:631-645.

25 Zhu H, Pi D, Leng W, Wang X, Hu CA, Hou Y, Xiong J, Wang C, Qin Q Liu Y: Asparagine preserves intestinal barrier function from LPS-induced injury and regulates CRF/CRFR signaling pathway. Innate Immun 2017;23:546-556.

-26 Baxt LA, Xavier RJ: Role of Autophagy in the Maintenance of Intestinal Homeostasis. Gastroenterology 2015;149:553-562.

-27 Jiang P, Guo Y, Dang R, Yang M, Liao D, Li H, Sun Z, Feng Q, Xu P: Salvianolic acid B protects against lipopolysaccharide-induced behavioral deficits and neuroinflammatory response: involvement of autophagy and NLRP3 inflammasome. J Neuroinflammation 2017;14:239.

-28 Nguyen HT, Lapaquette P, Bringer MA, Darfeuille-Michaud A: Autophagy and Crohn's Disease. J Innate Immun 2013;5:434-443.

29 Deretic V: Autophagy in immunity and cell-autonomous defense against intracellular microbes. Immunol Rev 2011;240:92-104.

30 Kuma A, Hatano M, Matsul M, Yamamoto A, Nakaya H, Yoshimori T, Ohsumi Y, Tokuhisa T, Mizushima N: The role of autophagy during the early neonatal starvation period. Nature 2004;432:1032-1036.

-31 Huang Y, Hua M, Cui X: Fungal $\beta$-Glucan Activates the NLRP3 Inflammasome in Human Bronchial Epithelial Cells Through ROS Production. Inflammation 2018;41:164-173. 


\section{Cellular Physiology Cell Physiol Biochem 2018;49:190-205 \begin{tabular}{l|l|l} 
and Biochemistry Published onlıne: 22 August 2018 & $\begin{array}{l}\text { (c) } 2018 \text { The Author(s). Published by S. Karger AG, Basel } \\
\text { www.karger.com/cpb }\end{array}$
\end{tabular}}

Feng et al.: Short-Chain Fatty Acids Protect Intestinal Barrier

32 Moon JS, Nakahira K, Chung KP, DeNicola GM, Koo MJ, Pabon MA, Rooney KT, Yoon JH, Ryter SW, StoutDelgado H, Choi AM: NOX4-dependent fatty acid oxidation promotes NLRP3 inflammasome activation in macrophages. Nat Med 2016;22:1002-1012.

33 Dalla-Pozza E, Manfredi M, Brandi J, Buzzi A, Conte E, Pacchiana R, Cecconi D, Marengo E, Donadelli M: Trichostatin A alters cytoskeleton and energy metabolism of pancreatic adenocarcinoma cells:An in depth proteomic study. J Cell Biochem 2018;119:2696-2707.

34 Cleophas MC, Crişan TO, Lemmers H, Toenhake-Dijkstra H, Fossati G, Jansen TL, Dinarello CA, Netea MG, Joosyen LA: Suppression of monosodium urate crystal-induced cytokine production by butyrate is mediated by the inhibition of class I histone deacetylases. Ann Rheum Dis 2016;75:593-600.

-35 Ba MC, Long H, Wang S, Wu YB, Zhang BH, Yan ZF, Yu FH, Cui SZ: Hyperthermia enhances radiosensitivity of colorectal cancer cells through ROS inducing autophagic cell death. J Cell Biochem 2018;119:3763-3774.

-36 Zhou Q Fu X, Wang X, Wu Q Lu Y, Shi J, Klaunig JE, Zhou S: Autophagy plays a protective role in Mn-induced toxicity in PC12 cells. Toxicology 2018;394:45-53.

37 Zaki MH, Boyd KL, Vogel P, Kastan MB, Lamkanfi M, Kanneganti TD: The NLRP3 Inflammasome Protects against Loss of Epithelial Integrity and Mortality during Experimental Colitis. Immunity 2010;32:379-391.

38 Qiao Y, Qian J, Lu Q Tian Y, Chen Q Zhang Y: Protective effects of butyrate on intestinal ischemiareperfusion injury in rats. J Surg Res 2015;197:324-330.

39 Chen G, Fu S, Feng W, Huang B, Xu S, Wang W, Liu J: AMP010014A09 in Sus scrofa encodes an analog of g protein-coupled receptor 109A, which mediates the anti-inflammatory effects of beta-hydroxybutyric acid. Cell Physiol Biochem 2017;42:1420-1430.

40 Macia L, Tan J, Vieira AT, Leach K, Stanley D, Luong S, Maruya M, Ian McKenzie C, Hijikata A, Wong C, Binge L, Thorburn AN, Chevalier N, Anq C, Marino E, Robert R, Offermanns S, Teixeira MM, Moore RJ, Flavell RA, et al.: Metabolite-sensing receptors GPR43 and GPR109A facilitate dietary fibre-induced gut homeostasis through regulation of the inflammasome. Nat Commun 2015;6:6734.

-41 Kanauchi O, Iwanaga T, Mitsuyama K, Saiki T, Tsuruta O, Noguchi K, Toyonaga A: Butyrate from bacterial fermentation of germinated barley foodstuff preserves intestinal barrier function in experimental colitis in the rat model. J Gastroenterol Hepatol 1999;14:880-888.

42 Alenghat T, Osborne LC, Saenz SA, Kobuley D, Ziegler CG, Mullican SE, Choi I, Grunberg S, Sinha R, WynoskyDolfi M, Snyder A, Giacomin PR, Joyce KL, Hoang TB, Bewtra M, Brodsky IE, Sonnenberg GF, Bushman FD, Won KJ, Lazar MA, Artis D: Histone deacetylase 3 coordinates commensal-bacteria-dependent intestinal homeostasis. Nature 2013;504:153-157.

43 Hasegawa H, Bissonnette RP, Gillings M, Sasaki D, Taniguchi H, Kitanosono H, Tsuruda K, Kosai K, Uno N, Morinaga Y, Imaizumi Y, Miyazaki Y, Yanagihara K: Induction of apoptosis by HBI-8000 in adult T-cell leukemia/lymphoma is associated with activation of Bim and NLRP3. Cancer Sci 2016;107:1124-1133.

44 Jessop F, Hamilton RF, Rhoderick JF, Shaw PK, Holian A: Autophagy deficiency in macrophages enhances NLRP3 inflammasome activity and chronic lung disease following silica exposure. Toxicol Appl Pharmacol 2016;309:101-110.

45 Wu J, Li X, Zhu G, Zhang Y, He M, Zhang J: The role of Resveratrol-induced mitophagy/autophagy in peritoneal mesothelial cells inflammatory injury via NLRP3 inflammasome activation triggered by mitochondrial ROS. Exp Cell Res 2016;341:42-53.

-46 Pavillard LE, Cañadas-Lozano D, Alcocer-Gómez E, Marín-Aguilar F, Pereira S, Roertson AAB, Muntane J, Ryffel B, Cooper MA, Quiles JL, Bullon P, Ruiz-Cabello J, Cordero MD: NLRP3-inflammasome inhibition prevents high fat and high sugar diets-induced heart damage through autophagy induction. Oncotarget 2017;8:99740-99756.

47 Chiu HW, Chen CH, Chang JN, Chen CH, Hsu YH: Far-infrared promotes burn wound healing by suppressing NLRP3 inflammasome caused by enhanced autophagy. J Mol Med 2016;94:809-819.

48 Zhang C, Yan J, Xiao Y, Shen Y, Wang J, Ge W, Chen Y: Inhibition of Autophagic Degradation Process Contributes to Claudin-2 Expression Increase and Epithelial Tight Junction Dysfunction in TNF- $\alpha$ Treated Cell Monolayers. Int J Mol Sci 2017;18:e157.

49 Dai J, Zhang X, Li L, Chen H, Chai Y: Autophagy Inhibition Contributes to ROS-Producing NLRP3-Dependent Inflammasome Activation and Cytokine Secretion in High Glucose-Induced Macrophages. Cell Physiol Biochem 2017;43:247-256. 